\title{
Análisis del Rendimiento Académico en Relación al Perfil de Ingreso del Alumnado e Identificación de Carencias Formativas en Materias Básicas de dos Grados de Ingeniería
}

\author{
Paulo Etxeberria*, Elisabete Alberdi, Isabel Eguia y Mª José García \\ Universidad del País Vasco / Euskal Herriko Unibertsitatea, Escuela de Ingeniería de Bilbao, \\ Paseo Rafael Moreno 'Pitxitxi', ㄲo 2, 48013 Bilbao, España. (e-mail: paulo.etxeberria@ehu.eus, \\ elisabete.alberdi@ehu.eus, isabel.eguia@ehu.eus, mariajose.garcialopez@ehu.eus) \\ * Autor a quien debe ser dirigida la correspondencia
}

Recibido Dic. 5, 2016; Aceptado Feb. 3, 2017; Versión final Mar. 30, 2017, Publicado Ago. 2017

\begin{abstract}
Resumen
Con el objeto de aportar al mejoramiento del rendimiento académico del alumnado de primer curso de dos grados de ingeniería que se imparten en la Escuela de Ingeniería de Bilbao se ha realizado un estudio pormenorizado de los resultados académicos obtenidos en cada asignatura en función del perfil de ingreso desde la implantación de los nuevos planes de estudio. Además en cada materia se ha realizado un análisis de las carencias formativas del alumnado utilizando encuestas sobre conocimientos al inicio de la docencia de cada asignatura y a su término que recogen, entre otras, las principales dificultades a las que se ha enfrentado el alumnado en el transcurso de la asignatura. Con la información obtenida en este estudio se han identificado los puntos débiles que servirán como base para el diseño de actividades formativas necesarias para compensar las carencias detectadas.
\end{abstract}

\section{Analysis of the Academic Achievement in Relation with the Profile of Entrance and Educational Deficiencies Identification in Basic Matters in two Degrees of Engineering}

\begin{abstract}
As a contribution to the improvement of the academic performance of first year students of two degrees offered at the Engineering School of Bilbao, a detailed study of the results obtained in each of the subjects based on the students' entrance profile since the new degree programs were initiated, has been conducted. In addition to that, an analysis of the weak areas that the students have in each subject has been performed. To accomplish this, tests at the beginning and at the end of the courses have been conducted, compiling in this way the main difficulties that students face during the development of the course. With the results obtained in this study, various weak points have been detected. These findings will serve to design academic activities that compensate the detected weaknesses.
\end{abstract}




\section{INTRODUCCIÓN}

Este trabajo se enmarca dentro de un proyecto de innovación educativa (PIE) financiado por la Universidad del País Vasco (UPV/EHU) con una duración de dos años. Dicho proyecto es el inicio de un proceso enfocado a la mejora de la enseñanza-aprendizaje (Eguia et al., 2012; Etxeberria et al., 2014) en primer curso del grado en Ingeniería Civil y del grado en Ingeniería de Tecnología de Minas y Energía impartidos en la Escuela de Ingeniería de Bilbao (EIB). Para la mayoría de estudiantes la transición de la educación secundaria a estudios universitarios resulta una situación agobiante (Brooks y DuBois, 1995; Pratt et al., 2000). Además de tener que hacer frente a nuevas situaciones personales, los estudiantes suelen tener que adquirir nuevos hábitos de estudio en un entorno de aprendizaje que es desconocido para ellos.

En la literatura se pueden encontrar numerosas investigaciones con el objeto de determinar los factores de éxito académico en la universidad, teniendo en cuenta la diversidad de los estudiantes que comienzan sus estudios universitarios. En diferentes trabajos se han estudiado, los distintos factores que predicen el rendimiento académico de estos estudiantes. Tanto en (McKenzie y Schweitzer, 2001) como en (Brooks y DuBois, 1995) se concluye que existe una correlación significativa entre las notas medias obtenidas en la educación secundaria y los resultados obtenidos en la universidad. En concreto, análisis realizados con estudiantes de ingeniería (Marra et al., 2012), donde se estudian los factores por los que los estudiantes abandonan sus estudios, concluyen que la preparación académica de entrada está relacionada con el grado de dificultad que encuentran para poder cursar adecuadamente sus estudios (cuanta menor preparación inicial, mayor dificultad encuentran).

Por el contrario en (Parker et al., 2004), se estudia el éxito académico universitario en relación con algunas dimensiones de la inteligencia emocional y de las habilidades sociales, deduciendo que estos factores predicen mejor el rendimiento académico que las notas medias obtenidas en la educación secundaria. La experiencia docente del profesorado de primer curso de diferentes ingenierías ha constatado que un elevado número de estudiantes presenta dificultades formativas motivadas por el salto cualitativo del bachillerato a la universidad, sobre todo en lo referente a las metodologías docentes y las estrategias de aprendizaje (Barbas et al., 2007; Rodríguez-Muñiz y Díaz, 2015).

En el pasado se han llevado a cabo diferentes aproximaciones para tratar de mejorar el salto de la educación secundaria al primer curso de los estudios de ingeniería, tales como ofertar cursos introductorios, materiales de estudio auxiliares en cada asignatura, tutorías especializadas por parte del profesorado o por alumnado de cursos superiores, etc. (Baillie, 1998). En (Nesbit et al., 2005; Frank et al., 2003) se presenta un curso basado en la evaluación, que introduce métodos propios de la ingeniería, como la estrategia de diseño y resolución de problemas. En lo que se refiere a los cursos introductorios, estos se han solido implementar bien en formato papel (Mayén y Rodríguez, 2008), bien en plataformas virtuales (Jarne et al., 2013). En todos los casos, resulta necesario que el centro universitario y su profesorado se impliquen directamente.

Tanto la Comisión de Calidad como las Comisiones de Grado de la EIB han detectado diferencias significativas en el rendimiento académico de primer curso con respecto a cursos superiores que van desde 15 a 30 puntos a medida que se avanza en el grado y han propuesto que se estudien acciones de mejora para disminuir estas diferencias. Como consecuencia, este trabajo tiene por objeto por una parte analizar el rendimiento académico en relación con el perfil de ingreso del alumnado y por otra identificar las posibles carencias formativas del alumnado en las áreas objeto de estudio con la finalidad de diseñar actividades voluntarias antes de cursar las asignaturas. De esta manera se pretende mejorar el rendimiento académico de dichas materias. Por ello se ha creado un grupo de trabajo integrado por profesorado de diversas áreas de conocimiento que imparten docencia en cuatro asignaturas de primer curso, común a los dos grados: Algebra y Geometría, Expresión Gráfica I, Informática y Expresión Gráfica II; con un total de 24 créditos.

\section{METODOLOGÍA}

Para la identificación de estas carencias se ha analizado la posible relación entre los resultados obtenidos en las diferentes asignaturas y el perfil de ingreso del alumnado. Este estudio se ha hecho a través de dos vías: análisis de los resultados académicos obtenidos en cada asignatura en relación al perfil de ingreso y análisis de las encuestas entregadas al alumnado al inicio y al final de la docencia de cada asignatura. Primeramente se ha realizado un estudio estadístico para evaluar la relación entre el rendimiento académico del alumnado de primer curso y su perfil de ingreso. Para ello se han analizado los resultados en las asignaturas de primer curso en los cursos académicos 2010-11, 2011-12 y 2012-13: un total de 3.400 registros de estudiantes por asignatura y curso académico. Los datos relativos al perfil de ingreso se han obtenido consultando los informes elaborados por la dirección de la EIB relativos a los grados objeto de estudio. 
De forma paralela se han diseñado dos encuestas adaptadas a las particularidades de cada materia; una se pasó antes de cursar la asignatura y la otra al finalizar la misma. El objetivo de la primera fue obtener la percepción del alumnado sobre el nivel de conocimientos de la asignatura previo al inicio de su docencia y su valoración sobre las posibles dificultades a las que suponían se enfrentaría en el desarrollo de la misma sobre unos temas que se les planteaba. De forma similar la segunda encuesta proporcionó datos de la percepción sobre los mismos ítems una vez cursada la materia. El rango de los ítems de las encuestas va desde 1 (muy mala) hasta 6 (muy buena). A continuación se muestra como ejemplo la encuesta inicial de la asignatura de Algebra y Geometría (figura 1)

\section{ENCUESTA INICLAL (Algebra Y Geometria)}

Titulación: 口CIVIL IMINAS Y ENERGIA

Procedencia: Bachillerato $\square \quad$ Ciclos formativos $\square$ Otras titulaciones $\square \quad$ Otros $\square$

En el caso de los alumnos de bachillerato. ¿Cuándo has cursado por última vex matemáticas?: $1^{\circ}$ de Bachillerato $\square \quad 2^{\circ}$ de Bachillerato $\square$

Valora el nivel de formación previa recibida en Matemáticas /1 (muy mala) 6 (muy buena):

\begin{tabular}{|l|l|l|l|l|l|l|l|}
\hline Nivel de la formación recibida en: & 1 & 2 & 3 & 4 & 5 & 6 & NS/NC \\
\hline Matemáticas I & & & & & & & \\
\hline Matemáticas II & & & & & & & \\
\hline Matemáticas Aplicadas a las Clencias Sociales & & & & & & & \\
\hline
\end{tabular}

¿En selectividad te examinaste de Matemáticas II? $\quad$ No $\quad \square$ Si

¿En selectividad te examinaste de Matemáticas Aplicadas a C. Sociales? $\square$ No

\section{Conocimientos concretos:}

Valora el nivel de la formación previa en los siguientes temas /1 (muy mala) 6 (muy buena):

\begin{tabular}{|c|c|c|c|c|c|c|c|}
\hline Conceptos básicos de: & 1 & 2 & 3 & 4 & 5 & 6 & NS/NC \\
\hline \multicolumn{8}{|c|}{$\begin{array}{l}\text { Cálculo matemático básico (fracciones, resolución de } \\
\text { ecuaciones, simplificaciones ...) }\end{array}$} \\
\hline \multicolumn{8}{|l|}{ Determinantes } \\
\hline \multicolumn{8}{|l|}{ Cálculo matricial } \\
\hline \multicolumn{8}{|c|}{ Sistemas de ecuaciones lineales } \\
\hline \multicolumn{8}{|l|}{ Vectores en el espacio } \\
\hline Geometria analítica & & & & & & & \\
\hline
\end{tabular}

Si has contestado que no a la pregunta anterior, ¿qué tipo de apoyo extra crees que necesitaras para seguir la asignatura de Algebra y Geometria?

$\square$ Asistencia a academia especializada en la materia

$\square$ Ayuda de profesor/a particular

- Consulta de bibliografía básica sobre la materia

口. Otros, Indicar cuáles:

Teniendo en cuenta tus conocimientos previos, ¿Cuáles crees que son las principales dificultades a las que te enfrentarás para seguir la asignatura de Algebra y Geometria?

ㅁ. Los conceptos aprendidos previamente no los tengo demasiado claros

- Tengo falta de destreza en el manejo del cálculo básico

- Observo carencia en el uso del lenguaje apropiado de la asignatura

$\square$ No he trabajado suficientemente la capacidad de razonamiento

$\square \quad$ El nivel de los ejercicios realizados con anterioridad es muy básico y escaso

$\square$ La metodologia de aprendizaje va a diferir bastante

Otras dificultades. Indicar cuales:

Fig. 1: Ejemplo de encuesta inicial (asignatura "Algebra y Geometría")

\section{RESULTADOS}

Los resultados del trabajo se presentan en dos bloques: por una parte se exponen los resultados académicos de las asignaturas en relación al perfil de ingreso y por otro se analizan las encuestas de percepción del alumnado con respecto a sus conocimientos sobre las áreas objeto de estudio. 


\section{Análisis de los resultados de las asignaturas en relación al perfil de ingreso}

El análisis de los resultados académicos (aprobado/suspenso) obtenidos por el alumnado de primer curso, en relación a diferentes variables que definen su perfil de ingreso, sirvió para confirmar algunos aspectos detectados por el profesorado tras varios años de docencia en primer curso y que ya han sido estudiados en otros trabajos (González, 1990; Rodríguez et al., 2004). En la figura 2 se muestran, a modo de ejemplo, los resultados para una asignatura concreta y un curso académico determinado.

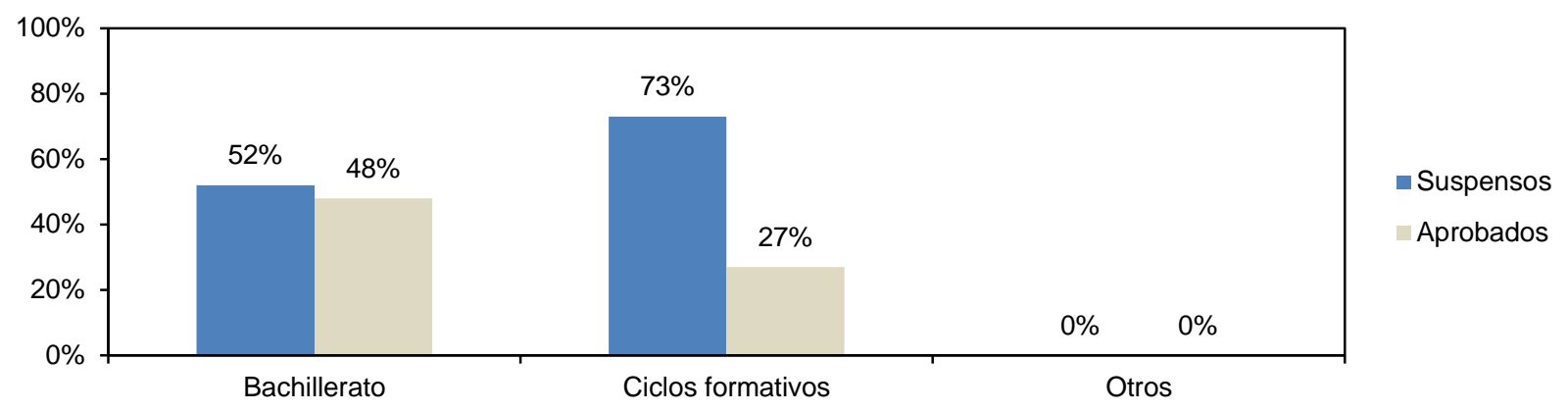

Fig. 2: Ejemplo de resultados -vs- perfil de ingreso en una asignatura (“Expresión Gráfica I”, curso 2010-11).

El alumnado proveniente de bachillerato obtiene mejores resultados que el procedente de ciclos formativos en prácticamente todas las asignaturas y cursos académicos. Estas diferencias son especialmente significativas en asignaturas con una alta componente matemática. En lo que se refiere al alumnado que proviene de bachillerato, los mejores resultados los obtienen aquellos que han cursado la especialidad "Ciencias y Tecnología". Hay que señalar que este alumnado es el mayoritario en los dos grados objeto de este trabajo (alrededor del 90\% del alumnado procede de bachillerato). En las distintas especialidades de los ciclos formativos no se ha encontrado ninguna relación estadísticamente fiable. Debe tenerse en cuenta que la muestra es muy inferior a la existente para alumnado de bachillerato ( 25 estudiantes de ciclos formativos frente a 110 de bachillerato aproximadamente todos los años).

Evidentemente, el alumnado con mejores notas de acceso obtiene mejores resultados, tanto los de bachillerato como los de ciclos formativos. Esta característica es común a todas las asignaturas y se repite en todos los cursos académicos. En la figura 3 se muestran los porcentajes de aprobados referentes a otra asignatura concreta.

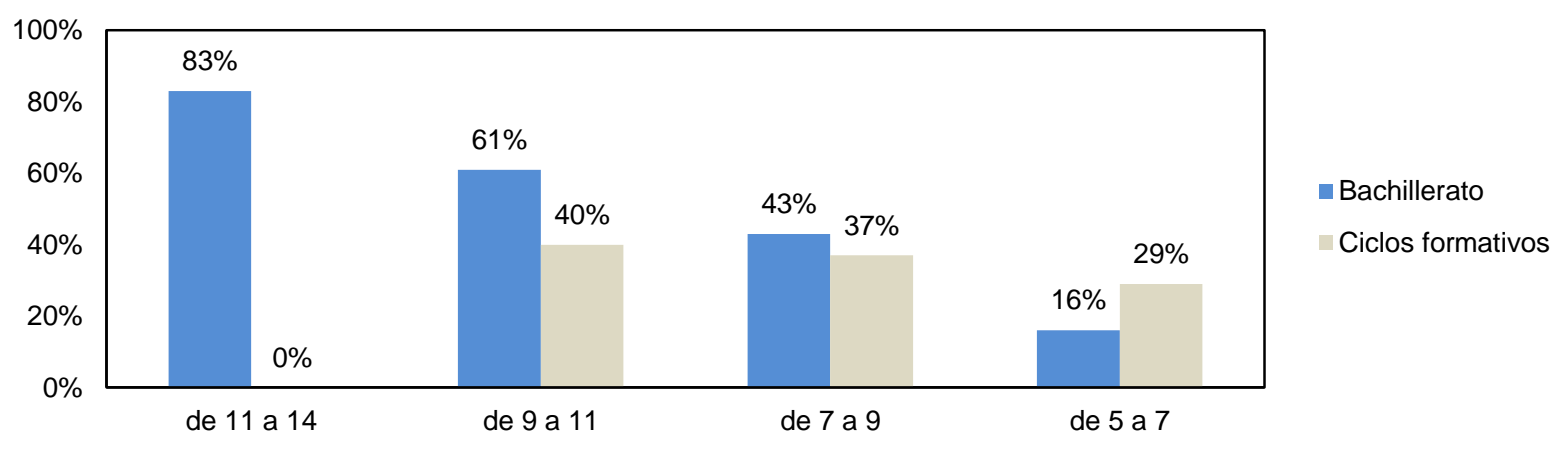

Fig. 3: Ejemplo de resultados (aprobados) -vs- perfil de ingreso en una asignatura (“Álgebra y Geometría”, curso 2012-13).

Se aprecian algunas diferencias entre los resultados obtenidos por el alumnado dependiendo de su procedencia (bachillerato o ciclos formativos). El primero obtiene mejores resultados que el segundo en asignaturas en las que se manejan conceptos abstractos y/o la base matemática es considerable (Expresión Gráfica I, Álgebra y Geometría). En asignaturas con menor abstracción o fundamentación matemática (Expresión Gráfica II e Informática) las diferencias son menores.

\section{Análisis de las encuestas}

El análisis de las encuestas iniciales y finales realizadas al alumnado ha permitido detectar aquellas áreas temáticas que conviene que el alumnado profundice previamente. Además, se ha obtenido información sobre las dificultades procedimentales observadas por el alumnado. Todo ello ha permitido diseñar contenidos teóricos y actividades prácticas que el alumnado, de manera voluntaria, podrá utilizar antes del 
inicio del curso académico. A continuación se muestra el análisis de las asignaturas que se estudian en este trabajo.

\section{Análisis de las encuestas realizadas para Álgebra y Geometría}

Las encuestas correspondientes a la asignatura "Álgebra y Geometría" se pasaron al comienzo y al final del primer cuatrimestre del curso 2012-13. De los resultados de las mismas se desprende que el $86 \%$ del alumnado de bachillerato ha cursado la asignatura "Matemáticas II" y el $92 \%$ del alumnado encuestado se examinó de esta materia en la prueba de acceso a la universidad (PAU). En la figura 4 se muestran los conocimientos previos que el alumnado posee sobre las diferentes áreas que se trabajan en la asignatura.

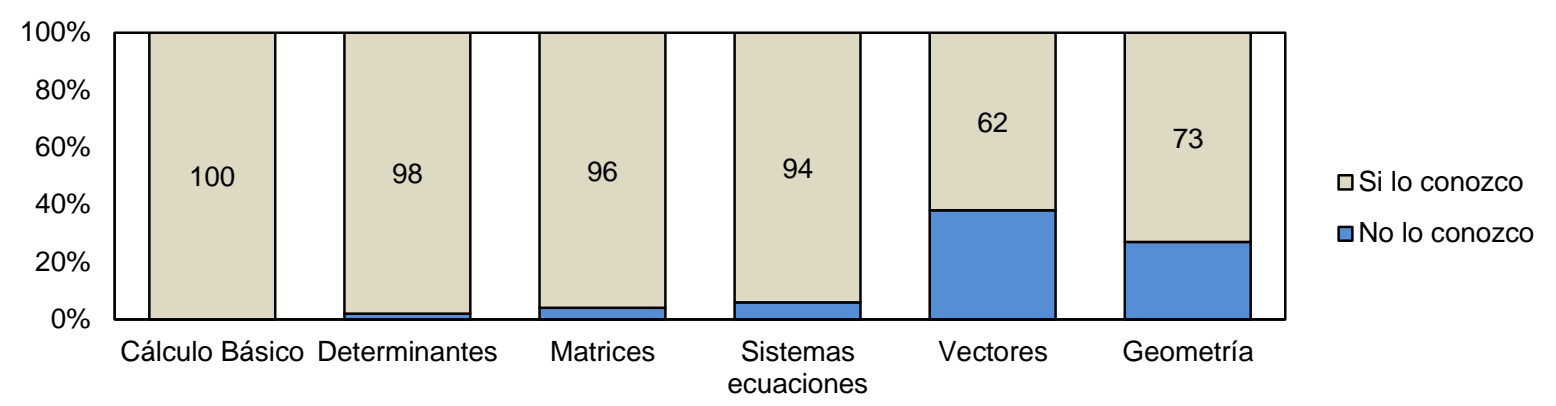

Fig. 4: Conocimientos previos del alumnado (asignatura "Álgebra y Geometría", curso 2012-13)

Se observa que la mayor parte del alumnado ha adquirido a lo largo de su formación preuniversitaria conocimientos de bastantes temas que se trabajan en la asignatura, siendo también significativo el porcentaje que desconoce un importante tema de la misma (Vectores en el espacio). Con el fin de conocer el nivel de formación previa del alumnado, se ha cuestionado al mismo su percepción del nivel que tiene de las diferentes áreas temáticas antes y después de haber cursado la asignatura. La percepción del alumnado sobre el nivel de conocimientos previos aparece representada en la figura 5.

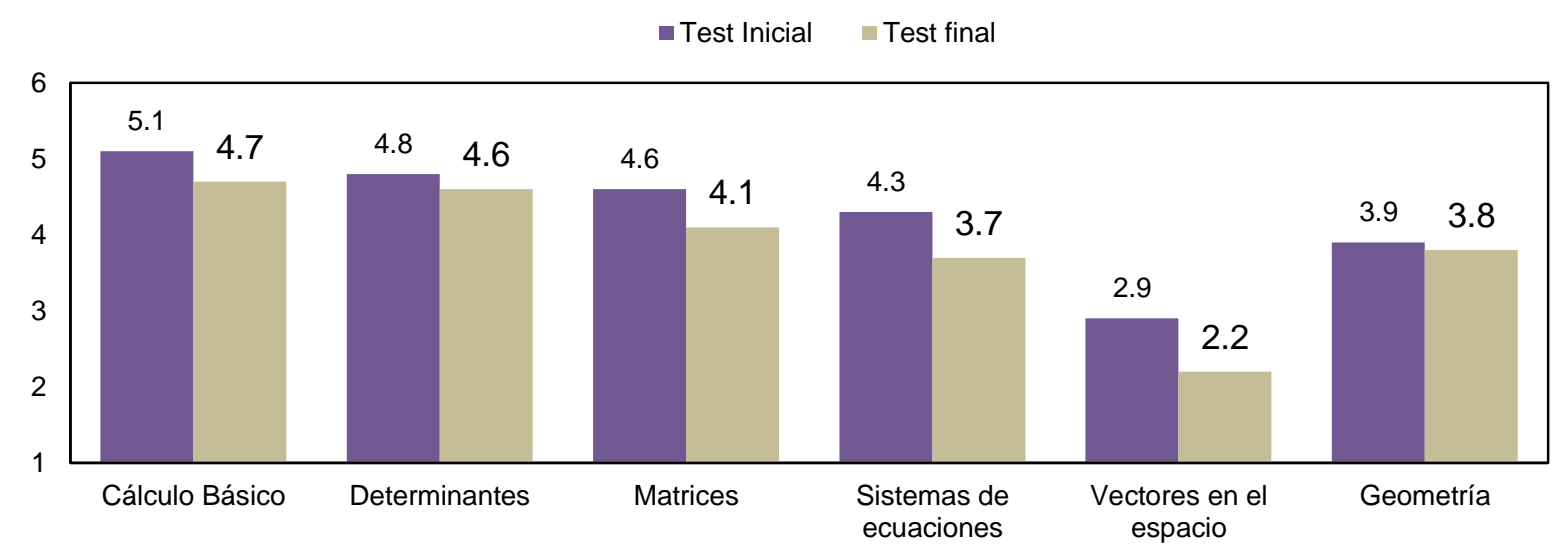

Fig. 5: Valoración de la formación previa en diferentes temas (Asignatura “Álgebra y Geometría”, curso 2012-13)

Como resultado se observa que el conocimiento que creen tener es bastante alto en la mayoría de los temas aunque la percepción es ligeramente menor una vez cursada la materia. Se constata que el nivel de formación previa que cree tener el alumnado es adecuado al contenido de la asignatura en muchas de sus áreas. Otro aspecto importante de este estudio es destacar las principales dificultades que el alumnado ha encontrado para seguir la asignatura. En concreto ha observado falta de destreza en el manejo del cálculo básico y en su capacidad de razonamiento.

\section{Análisis de las encuestas realizadas para Informática}

Las encuestas correspondientes a la asignatura "Informática" se pasaron al comienzo y al final del segundo cuatrimestre del curso 2012-13. De los resultados de las encuestas se desprende que el porcentaje de alumnos que han cursado informática en el bachillerato y los que no es el mismo (46\%). El conocimiento de la asignatura a nivel global es valorado por los estudiantes con un 3. No obstante, esta valoración disminuye cuando se les pregunta sobre temas concretos de la asignatura como se demuestra en la figura 6. 


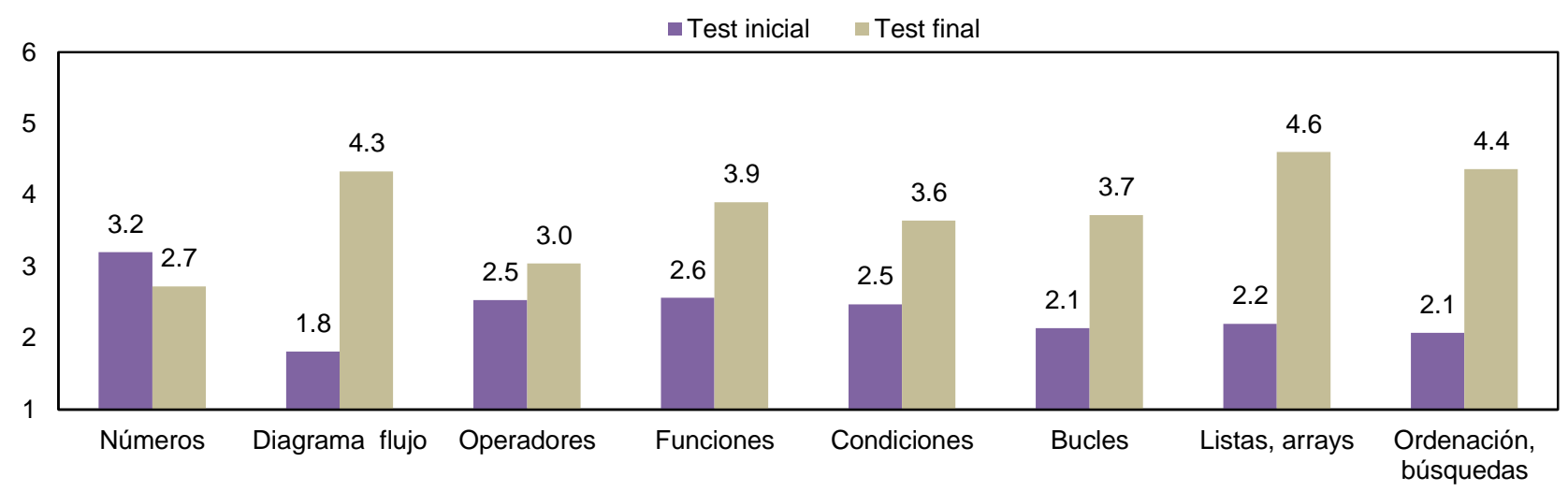

Fig. 6: Valoración de la formación previa (test inicial) / nivel de dificultad percibido (test final) (Asignatura "Informática", curso 2012-13)

Una vez cursada la asignatura se evaluó el nivel de dificultad que para el alumnado habían tenido aquellos temas cuestionados en el test inicial sobre la valoración de su formación y que aparecen relejados en la figura anterior. Se observa que la percepción del alumnado sobre su formación antes de cursar la asignatura se corresponde con los niveles de dificultad que han encontrado (por ejemplo representación de números y diagramas de flujo de forma inversa). La inmensa mayoría del alumnado no ha necesitado ningún tipo de apoyo para seguir adecuadamente la asignatura. La principal dificultad encontrada ha sido la realización de los diagramas de flujo.

Dado que la utilización de lenguajes de programación es fundamental en el desarrollo de la asignatura, además del análisis de los temas citados anteriormente, se estudió su conocimiento previo. Se comprobó que más de las tres cuartas partes del alumnado no han utilizado nunca los lenguajes de programación lo que ha influido en su falta de destreza en programación.

\section{Análisis de las encuestas realizadas para Expresión Gráfica I}

Las encuestas correspondientes a la asignatura "Expresión Gráfica I" se pasaron al comienzo y al final del primer cuatrimestre del curso 2012-13. De los resultados de las encuestas se desprende que el $64,3 \%$ del alumnado de bachillerato ha cursado las asignaturas "Dibujo técnico I" y "Dibujo técnico II". Algunos han cursado solo una de ellas y el $14,3 \%$ ninguna. No obstante el $82 \%$ del alumnado encuestado se presentó a la materia "Dibujo técnico II" en la PAU. En esta asignatura se abordan dos sistemas de representación claramente diferenciados de aplicación en la ingeniería: Sistema Diédrico y Sistema de Planos Acotados. En general una vez cursada la asignatura, la percepción del alumnado sobre la formación previa recibida disminuye prácticamente en todos los temas correspondientes al Sistema Diédrico (figura 7). Esta diferencia es mayor en el caso del primer tema posiblemente porque es el primero que se aborda en el curso.

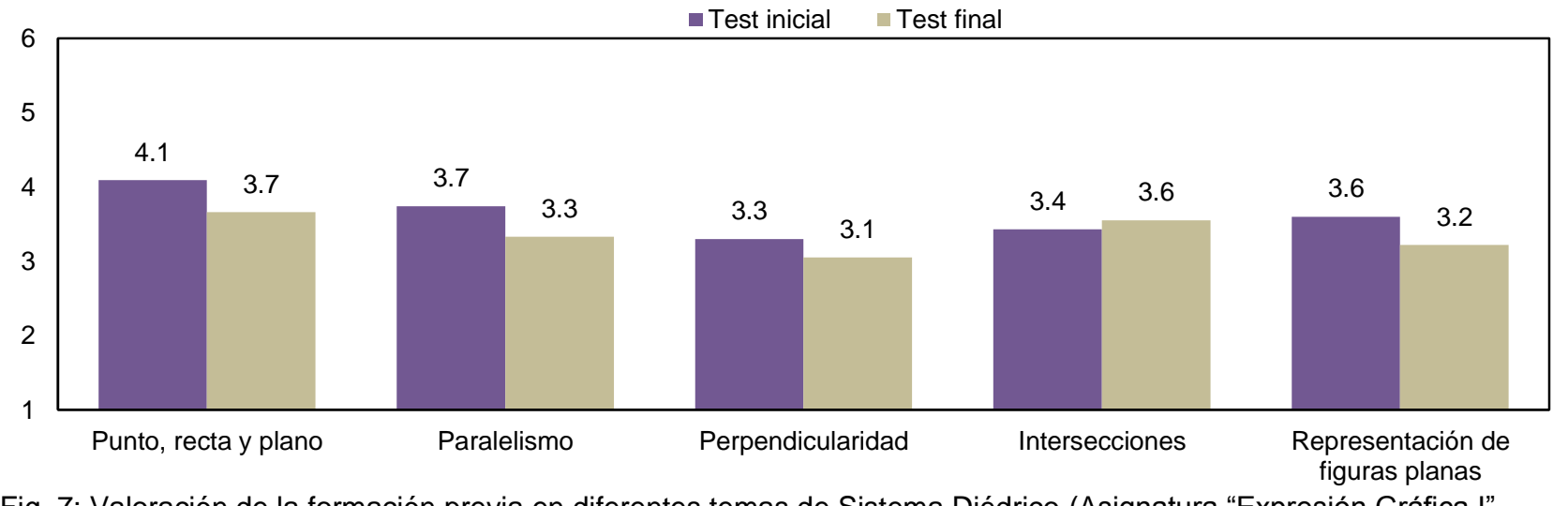

Fig. 7: Valoración de la formación previa en diferentes temas de Sistema Diédrico (Asignatura "Expresión Gráfica l", curso 2012-13)

En el caso del Sistema de Planos Acotados la diferencia entre las valoraciones en los test inicial y final es sustancial, probablemente por el desconocimiento generalizado de dicho sistema. Al comparar los dos bloques en los que se divide la asignatura se constata que el conocimiento sobre el Sistema Diédrico es mucho mayor que el del Sistema de Planos Acotados, hecho que ya había sido percibido por el equipo docente de la asignatura. El 72\% del alumnado encuestado considera que los conocimientos previos no han sido suficientes para llevar de forma 
adecuada la asignatura por lo que un $33 \%$ ha necesitado algún tipo de ayuda para seguirla. En cuanto a las principales dificultades encontradas para cursar la asignatura, un $33 \%$ considera que el nivel de los ejercicios realizados antes de cursar el grado es muy básico o escaso y un $29 \%$ señala que la metodología de aprendizaje es muy diferente.

\section{Análisis de las encuestas realizadas para Expresión Gráfica II}

Las encuestas correspondientes a la asignatura "Expresión gráfica II" se pasaron al comienzo y al final del segundo cuatrimestre del curso 2012-13. Como en el caso anterior la mayoría del alumnado de bachillerato ha cursado las asignaturas "Dibujo técnico I" y "Dibujo técnico II". El nivel de formación adquirida en dichas asignaturas es, en opinión del alumnado consultado, de 3,87 y 3,80 respectivamente. La mayoría de los estudiantes tanto de bachillerato como de ciclos formativos, ha estudiado previamente, con mayor o menor profundidad, los temas que se desarrollan en la asignatura. Los temas que prácticamente todo el alumnado ha estudiado son los relacionados con vistas, acotación y perspectivas; los menos conocidos (66\%-74\%) son "Dibujo de ingeniería" y "Dibujo por ordenador (CAD)". En cuanto al nivel de dificultad percibido en los temas mencionados anteriormente, los resultados son bastante homogéneos: un mínimo de 3,0 (CAD 2D) y un máximo de 4,0 (Croquización) (figura 8).

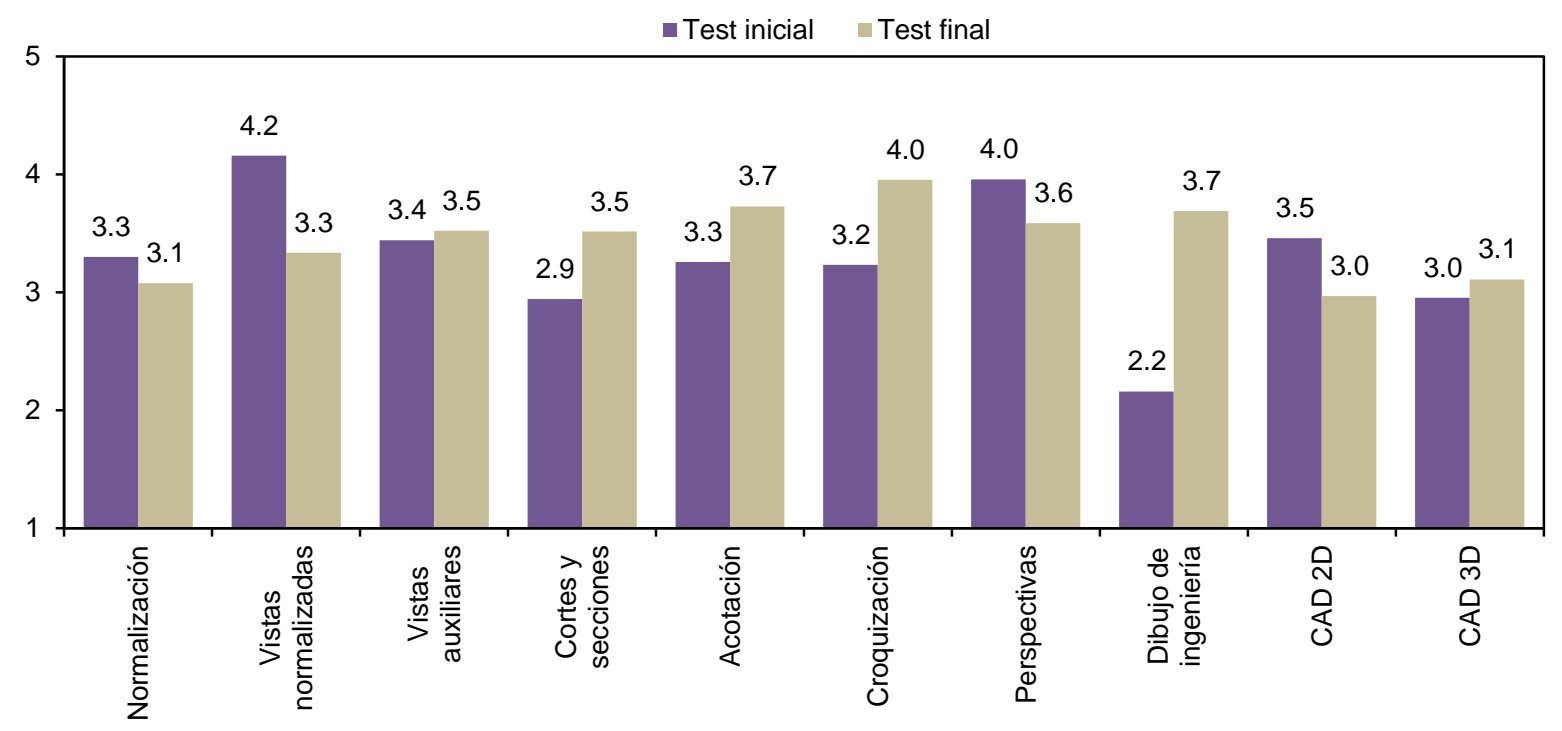

Fig. 8: Valoración de la formación previa (test inicial) / nivel de dificultad percibido (test final) (Asignatura "Expresión Gráfica II", curso 2012-13)

\section{CONCLUSIONES}

El alumnado que ha cursado bachillerato obtiene mejores resultados que el que ha cursado ciclos formativos en prácticamente todas las asignaturas y cursos académicos, siendo éstos aún mejores en asignaturas con un grado importante de abstracción tales como Álgebra y Geometría y Expresión Gráfica I.

En lo que se refiera al alumnado que proviene de bachillerato, los mejores resultados los obtiene el alumnado que ha cursado la especialidad "Ciencias y Tecnología".El alumnado con mejores notas de acceso obtiene mejores resultados (tanto de bachillerato como de ciclos formativos). Este hecho ocurre en todas las asignaturas y en todos los cursos académicos.La mayoría de los estudiantes han trabajado previamente, aunque sea a nivel básico, gran parte de los temas de las asignaturas, con algunas excepciones como Espacios Vectoriales en Álgebra y Geometría, Sistemas de Planos Acotados en Expresión Gráfica I o Dibujo de Ingeniería en Expresión Gráfica II. Este hecho es claramente diferente en el caso de la asignatura Informática, cuyo nivel de conocimiento previo es muy escaso aunque no determinante en sus resultados académicos.

El alumnado de Expresión Gráfica I manifiesta que sus conocimientos previos no han sido suficientes para abordar la asignatura con éxito. Este caso no ocurre en el resto de asignaturas. La información suministrada por las encuestas de asignatura y el análisis de la relación entre el perfil de ingreso y los resultados obtenidos, unido a la experiencia del profesorado, ha permitido diseñar un conjunto de actividades de profundización con el proyecto de proponer un curso cero virtual que sirva de puente entre los estudios secundarios y los estudios universitarios en el ámbito de la ingeniería. 


\section{REFERENCIAS}

Baillie, C., Addressing First-year Issues in Engineering Education, European Journal of Engineering Education, 23 (4), 453-465 (1998)

Barbas, F.J., Fernández, C. y otros tres autores, El curso de conocimientos previos y la acción tutorial: reflexiones desde la práctica, REOP-Revista Española de Orientación y Psicopedagogía, 18(1), 59-64 (2007)

Brooks, J.H. y DuBois, D.L., Individual and environmental predictors of adjustment during the first year of college, Journal of College Student Development, 36(4), 347-360 (1995)

Eguia, I., García, M.J. y otros dos autores, Análisis y reflexiones de los resultados académicos obtenidos en una actividad interdisciplinar de dos asignaturas básicas en el ámbito de la geometría. Actas del XX Congreso Universitario de Innovación Educativa en las Enseñanzas Técnicas, Las Palmas de Gran Canaria (2012)

Etxeberria, P., De Luis, A. y otros ocho autores, Análisis del rendimiento académico en las materias básicas de primer curso de dos Grados en Ingeniería en relación con el perfil de ingreso del alumnado, Actas del XXII Congreso Universitario de Innovación Educativa en las Enseñanzas Técnicas, Almadén (2014)

Frank, M., Lavy, T., Elata, D., Implementing the project-based learning approach in an academic engineering course, International Journal of Technology and design Education, 13(3),273-288 (2003)

González, R.M., La Investigación educativa sobre la Universidad, Principales dificultades en el rendimiento académico en el primer año de carreras de Ingeniería, p. 261, CIDE, Madrid (1990)

Jarne, G., Zabal, M.T., Minguillón, E., Autoaprendizaje de las matemáticas con nuevas tecnologías en los grados de carácter económico, e-Pública, 13, p. 1 (2013)

Marra, R.M., Rodgers, K. A. y otros dos autores, Leaving Engineering: a multi-year single institution study, Journal of Engineering Education, 101(1), 6-27 (2012)

Mayén, M. y R. Rodríguez, Introducción a la Química Universitaria. Curso Cero, Servicio de Publicaciones de la Universidad de Córdoba (2008)

McKenzie, K. y Schweitzer, R., Who succeeds at University? Factors predicting academic performance in first year Australian university students, Higher Education Research \& Development, 20(1), 21-33 (2001)

Nesbit, S.M., Hummel, S., R., Piergiovanni, P.R., Schaffer, J.P., A design and assessment-based introductory engineering course, International Journal of Engineering Education, 21(3), 434-445 (2005)

Parker, J.D.A., Summerfeldt, L.J., Hogan, M.J., Majeski, S.A., Emotional intelligence and academic success: examining transition from high school to university, Personality and Individual Differences, 36 (1), 163-172 (2004)

Pratt, M. W., Hunsberger, B. y otros ocho autores, Facilitating the transition to university: evaluation of a social support discussion intervention program, Journal of College Student Development, 41(4), 427-441 (2000)

Rodríguez-Muñiz, L.J. y P. Díaz, Estrategias de las universidades españolas para mejorar el rendimiento en matemáticas del alumnado de nuevo ingreso, Aula Abierta, 43(2), 69-76 (2015)

Rodríguez, S., Fita, E., Torrado, M., El rendimiento académico en la transición secundaria-universidad, Revista de Educación, 334, 391-414 (2004) 